\title{
Actitudes de aculturación, sensibilidad intercultural y prejuicio en población autóctona e inmigrante en España
}

Antonio GonZÁlez LÓpez

Centro Asociado Universidad de Educación a Distancia (Cuenca-España)

María Paz Ramírez López

Universidad Pontificia Comillas (España)

\section{Introducción}

En el momento histórico actual, determinados fenómenos característicos del siglo XXI como la comunicación, las relaciones grupales, la diversidad o las migraciones se constituyen en fenómenos globales generadores de una cultura universal con importantes repercusiones en los contextos locales donde en último término se manifiestan. Con respecto a las migraciones, en torno al $3 \%$ de la población mundial es considerada migrante o persona que vive en otro país al de su nacimiento como consecuencia de factores demográficos, desigualdades económicas, conflictos sociales y políticos o liberalización del comercio y de la mano de obra (International Organization for Migration, 2012). Las sociedades contemporáneas pueden ser consideradas como plurales, con numerosos grupos culturales, étnicos y religiosos que intentan vivir entre sí, relacionándose en un mismo espacio común (Labedeva, Tatarko, \& Berry, 2016). En el caso concreto del contexto español, 
los estudios de población reflejan que más de cinco millones de personas nacidas en otros países viven dentro de nuestras fronteras. De esta forma, en España, en torno al 10 \% de su población censada es inmigrante (Instituto Nacional de Estadística, 2014), lo que nos convierte en un país receptor de personas que llegan desde más allá de nuestras fronteras (Arango, 2000).

Las tendencias en migración internacional están sufriendo un rápido proceso de cambio en su alcance y en sus objetivos, debido principalmente a cuestiones sociales, medioambientales, políticas y económicas. Los países occidentales, y más en concreto la Unión Europea, intentan evitar la creciente afluencia de emigrantes ilegales no cualificados que llegan en busca de oportunidades económicas y de mejora del nivel de vida o escapando de situaciones de pobreza extrema y de conflictos bélicos o políticos. Estos grupos de migrantes son percibidos por la población de los países receptores como un problema para su identidad nacional, sus opciones de trabajo, su seguridad y sus servicios sociales, de salud y educativos. Sin embargo, de forma simultánea, estos mismos países están necesitados de mano de obra cualificada y por tanto buscan atraer y reubicar en sus territorios a nuevos grupos con altas destrezas laborales, necesarias para competir en las economías de la sociedad del conocimiento. Es por ello que las actitudes que emergen en la población de las sociedades de acogida vienen muy influidas por sus condiciones económicas, políticas y sociales y en particular por los contextos sociales donde se sitúan los inmigrantes al establecerse en los países receptores (Esses, Wagner, Wolf, Preiser, \& Wilbur, 2006).

Ante este estado de cosas, los procesos migratorios se han convertido en un fenómeno estructural con el que las sociedades tienen que aprender a convivir. La situación global de diversidad étnica y cultural está provocando importantes cambios socioculturales que obligan a entender la influencia que los contextos sociales ejercen en los modos de convivencia y en el bienestar psicológico, ambos aspectos son necesarios para la integración entre las sociedades receptoras y los grupos de personas migrantes (Portes, Celaya, Vickstrom, \& Aparicio, 2012; Hui, Chen, Leung, \& Berry, 2015). Es por esto que los investigadores sociales han de asumir el compromiso de generar 
nuevos conceptos explicativos de estos fenómenos y desarrollar un adecuado marco teórico e instrumentos de conocimiento acordes al contexto donde ocurren los procesos migratorios.

\section{Marco teórico}

Las interacciones humanas transnacionales han puesto en primer plano una serie de conceptos críticos en este campo de estudio como son el 'multiculturalismo', la 'diversidad cultural' y la 'aculturación'. El término aculturación ya fue definido en la primera mitad del siglo XX como "aquellos fenómenos que resultan cuando grupos de individuos con diferentes culturas entran en contacto continuo, con cambios posteriores en los patrones culturales originales de uno o ambos grupos" (Redfield, Linton, \& Herskovits, 1936: 149). De acuerdo a la importancia de este concepto, desde las ciencias sociales se han desarrollado numerosos modelos teóricos para evaluar el proceso de aculturación y los cambios en los valores e identidad cultural que se generan entre grupos de inmigrantes y la sociedad receptora (Berry, 1980; Padilla, 1980; Bourhis, Moïse, Perreault, \& Senécal, 1997; Piontkowski, Rohmann, \& Florack, 2002; Navas, García, Sánchez, Rojas, Pumares, \& Fernández, 2005). De esta forma, la aculturación ha sido analizada por los distintos modelos teóricos en relación con el ajuste sociocultural y la adaptación psicológica a un nuevo contexto sociocultural (Ward \& Kennedy, 1994) y también a variables psicosociales implicadas en las relaciones grupales (Padilla \& Perez, 2003).

En concreto, el Modelo de Aculturación de Berry (1980) plantea que la aculturación sucede a través de un proceso caracterizado por el contacto, el conflicto y la adaptación. A lo largo de este proceso adaptativo se dan cuatro respuestas u opciones de aculturación según el valor que los grupos minoritarios conceden a mantener su propia herencia cultural y al valor otorgado a relacionarse con la cultura de acogida. Estas respuestas adaptativas son la 'integración', la 'asimilación', la ‘separación' y la 'marginación'. La integración se refiere al mantenimiento de la cultura de origen a la vez que se produce una 
apertura a la sociedad de acogida. La asimilación se da cuando se abandona la cultura de origen y se llevan a cabo relaciones con la sociedad de acogida. La separación sucede cuando se mantiene la identidad cultural de origen y no hay relación con la sociedad receptora y, finalmente, la marginación ocurre cuando los inmigrantes abandonan su cultura de origen y además no establecen contacto con la sociedad de acogida. A este respecto, numerosos trabajos concluyen que la integración es la estrategia adaptativa preferida por los inmigrantes y la que se relaciona con mayores niveles de salud mental y con mejores actitudes intergrupales (Berry, 1997; Pfafferott \& Brown, 2006). Sin embargo, este modelo de categorías de aculturación ha sido criticado por asumir que las cuatro respuestas de aculturación tienen la misma validez, de tal forma que se ha llegado a plantear la posible existencia de opciones de aculturación propias de cada individuo (Bourhis, Moïse, Perreault, \& Senécal, 1997; Rudmin, 2009).

Por su parte, el Modelo Ampliado de Aculturación Relativa (Navas et al., 2005) hace referencia a dos planos de aculturación. El plano real referido a las estrategias de aculturación que eligen los inmigrantes y que los autóctonos perciben que los inmigrantes ponen en práctica; y el plano ideal referente a las actitudes u opciones de aculturación preferidas por inmigrantes y que los autóctonos prefieren para ellos. Desde este modelo se concluye que el proceso adaptativo es complejo y relativo pues se dan diferentes estrategias y distintas actitudes dependiendo de que la interacción entre inmigrantes y autóctonos se produzca en unos u otros ámbitos de la realidad sociocultural. Estos ámbitos son el político -sistema político y de Gobierno-, laboral-procedimientos del trabajo-, económico -hábitos de consumo y economía familiar-, social-relaciones sociales y amistades-, familiar, religioso y, finalmente, el ámbito de las formas de pensar, donde se encuadran los principios y los valores (Navas et al., 2005).

Determinados trabajos de investigación concluyen que hay una escasa correspondencia entre las opciones de aculturación de los inmigrantes y la percepción de dichas elecciones por parte de los autóctonos, debido a que estas suelen estar sesgadas por actitudes negativas hacia el grupo inmigrante (Van Oudenhoven, Prins, \& Buunk, 1998; Zagefka \& Brown, 2002). En concreto, la discriminación o el prejuicio, 
entendido como una actitud negativa hacia un grupo social o hacia sus miembros (Allport, 1954; Brown, 1995), se constituyen en sesgos que condicionan las preferencias y percepciones del proceso de aculturación de los grupos de inmigrantes por parte de la sociedad de acogida (Briones, Verkuyten, Cosano, \& Tabernero, 2012). A este respecto, algunos trabajos han encontrado que, tanto en poblaciones mayoritarias como en los grupos minoritarios o de inmigrantes, las puntuaciones más bajas en prejuicio hacia el exogrupo se relacionan de forma positiva con la preferencia por la integración y las puntuaciones más altas con la preferencia por la marginación (Navas, García, Rojas, Pumares, \& Cuadrado, 2006; Zick, Wagner, Van Dick, \& Petzel, 2001). En la misma línea, la concordancia o discordancia entre la sociedad de acogida y los grupos de inmigrantes en las preferencias sobre las opciones de aculturación influiría en sus relaciones intergrupales, en los posibles conflictos e incluso en la elección de unas u otras opciones de aculturación por parte del grupo minoritario (Kosic, Mannetti, \& Sam, 2005; Piontkowski, Rohmann, \& Florack, 2002).

Por todo lo anterior, para entender el proceso de adaptación social y la convivencia entre grupos culturales es necesario considerar la influencia que las actitudes del grupo mayoritario ejercen sobre la forma en que las minorías culturales afrontan su proceso de aculturación. En este punto cabe destacar las aportaciones del multiculturalismo entendido como proceso de interacción y adaptación, a través de un intercambio social entre los diferentes grupos, basado en la negociación y la resolución de los conflictos, que desencadena cambios de la estructura social beneficiosos para los grupos culturales en contacto (Rodríguez-García, 2010).

Con el objetivo de generar actitudes de entendimiento entre diferentes grupos culturales que entran en contacto, hay que tener en cuenta la denominada competencia comunicativa intercultural, en cuanto conjunto de conocimientos, destrezas y actitudes interculturales necesarias para comunicarse y convivir de forma apropiada en sociedades caracterizadas por la diversidad cultural (Chen, 1990; Gudykunst, Wiseman, \& Hammer, 1977; Ruben \& Kealy, 1979). A este respecto, el Modelo de Desarrollo de la Sensibilidad Intercultural (Bennett, 1993) plantea que la convivencia entre culturas se basa en 
la sensibilidad intercultural definida como un cambio afectivo, cognitivo y conductual que iría desde posiciones etnocéntricas a posiciones etnorelativas de reconocimiento y apreciación de las diferencias culturales. Este modelo asume que conforme se experimentan diferencias culturales más complejas, se incrementa la competencia para las relaciones interculturales a través de un proceso de transformación basado en seis fases o etapas. Estas fases son: 1. Negación, donde se niegan las diferencias culturales importantes y se concibe la propia cultura como la única verdadera; 2. Defensa, cuando las diferencias culturales se viven como amenazantes y se considera la propia cultura como la única viable; 3. Minimización, donde para proteger la propia cosmovisión se resaltan las similitudes culturales enmascarando el reconocimiento y apreciación de las diferencias; 4. Aceptación, cuando se reconocen y aprecian las diferencias y similitudes de la propia y de otras culturas; 5. Adaptación, sucede cuando la persona puede cambiar su perspectiva cultural, mostrar empatía con las diferencias culturales y modificar los afectos e identidad cultural sobre la base de las diferencias culturales y; 6. Integración, cuando la propia experiencia del yo incluye las diferentes visiones culturales.

Por su parte, el Modelo de Competencia Comunicativa Intercultural (Chen \& Starosta, 1996) entiende la convivencia entre culturas por medio de la construcción de un multiculturalismo sustentado en la integración de las diferencias culturales y en la interacción cultural respetuosa. Según este modelo, la comunicación intercultural consta de tres componentes: 1 . La sensibilidad intercultural o aspecto afectivo, referida al deseo emocional de reconocer, aceptar y apreciar las diferencias culturales en situaciones de interacción intercultural; 2 . La conciencia intercultural o dimensión cognitiva, relativa al proceso de entendimiento de las convenciones culturales y de las similitudes-diferencias respecto a otra cultura; y 3. La competencia intercultural o aspecto conductual, referida a las habilidades de consecución de las metas comunicativas al interactuar con personas de otras culturas. De esta forma, la sensibilidad intercultural, en cuanto a componente afectivo, se entiende como la capacidad personal para desarrollar una emoción positiva hacia el entendimiento y aprecio de las diferencias culturales (Chen \& Starosta, 1996). 
Con base en el marco teórico mencionado y las categorías conceptuales que se han apuntado, el principal objetivo de los trabajos que se presentan a continuación consistió en estudiar las relaciones existentes entre las opciones de aculturación, la sensibilidad intercultural y el prejuicio en muestras de la población autóctona y de la población inmigrante latinoamericana de la ciudad de Cuenca (España). En concreto, este colectivo de inmigrantes latinoamericanos en la ciudad de Cuenca representa un $3 \%$ de la población censada según los datos del Padrón Municipal del año 2011, lo que supone un total de 1.702 personas inscritas en el padrón municipal como personas inmigrantes de origen latinoamericano. Desde esta línea y siguiendo los trabajos de Berry (1997), Piontkowski, Rohmann, \& Florack (2002) y Navas, Rojas, \& García (2011), se identificaron las opciones de aculturación de inmigrantes y autóctonos tanto en el plano ideal o de las actitudes como en el real o de las estrategias, así como las relaciones existentes entre la aculturación y el prejuicio manifestado por ambos grupos de población. Por otro lado, se analizaron las actitudes multiculturales y de aculturación de las distintas muestras en relación con la sensibilidad intercultural y con el prejuicio entendido como una actitud negativa hacia un grupo social o hacia sus miembros.

Los resultados de los trabajos que se presentan a continuación se convierten en un primer intento de elaborar un modelo de adaptación sociocultural de la población inmigrante en un contexto local específico, poniendo en relación una serie de variables como son las respuestas de aculturación en cuanto actitudes adaptativas a un nuevo contexto cultural, el prejuicio como actitud social de discriminación y la sensibilidad intercultural como actitud hacia las interacciones culturales y hacia la diversidad étnica (González \& Ramírez, 2016). El marco teórico y metodológico utilizado en estos trabajos de investigación se encuadra dentro de la línea de investigación en aculturación que considera la integración constructiva como un resultado de la competencia intercultural y del etnorelativismo (Bosky, 2008; Bennett, 1993). 


\section{Marco metodológico}

\section{Participantes}

Un total de 640 personas fueron encuestadas como participantes de estos trabajos de investigación. La muestra se distribuyó en 285 inmigrantes y 355 autóctonos. El trabajo de campo se llevó a cabo entre 2012 y 2013 . Encuestadoras entrenadas al efecto entregaron cuestionarios con las instrucciones precisas parar ser cumplimentados en ese mismo momento de forma individual y anónima en dependencias de ONG, de Asociaciones de Inmigrantes y de centros de trabajo públicos y privados de la ciudad de Cuenca (España), donde se solicitaba a los presentes, mayores de edad, su participación en estos estudios de investigación a través de la cumplimentación de dos cuestionarios con las instrucciones precisas para ser rellenados. En ambos casos, por tanto se siguió un muestreo no probabilístico. A todos los sujetos a los que se hizo entrega del cuestionario lo cumplimentaron y entregaron en ese mismo momento.

En el primer estudio, con respecto a la muestra de inmigrantes, 88 fueron hombres y 92 mujeres, siendo la media de edad 35.88 años (DT $=10.88)$ y su media de tiempo de estancia en España de 7.65 años $(\mathrm{DT}=3.72)$. Por lo que respecta a la muestra de autóctonos, en este caso todos docentes españoles, 92 fueron hombres y 142 mujeres, siendo la media de edad 45.46 años (DT=9.12) y la media de tiempo ejerciendo la enseñanza de 20.36 años (DT=10.16).

En el segundo estudio, con respecto a las muestras de inmigrantes, 44 fueron hombres y 61 mujeres, siendo la media de edad 36.34 años (DT $=10.42$ ) y su media de tiempo de estancia en España de 8.08 años (DT=4.03). Por lo que respecta a la muestra de autóctonos, 55 fueron hombres y 65 mujeres, siendo la media de edad 41.14 años (DT= 12.75). 


\section{Instrumento}

En ambos trabajos se construyó un doble cuestionario dirigido tanto a la población autóctona como a la población inmigrante. Las variables contempladas en los diferentes cuestionarios fueron las siguientes:

1. Características sociodemográficas. Género, edad, estudios realizados, estado civil, situación laboral, creencias políticas y frecuencia de relación con personas de otros países.

2. Actitudes de aculturación. Está variable se basó en las dimensiones del Modelo de Berry (1997) sobre la importancia o no concedida a la conservación de la identidad cultural de origen, y sobre la importancia o no de relacionarse con la sociedad de acogida. De esta forma, se elaboró una medida dicotómica para comprobar las preferencias sobre aculturación de los autóctonos para los inmigrantes por medio de dos cuestiones formadas por doble opción de respuesta donde solo se podía elegir una de las opciones. Se miden las opciones de aculturación basadas en las actitudes y estrategias hacia el mantenimiento de la cultura y hacia el contacto con la sociedad de acogida. De este modo, se evalúan los efectos de ambas dimensiones de forma separada, lo que permite clasificar a los participantes en cada una de las cuatro opciones de aculturación. Es decir, se miden las opciones reales y preferidas por los inmigrantes y aquellas opciones percibidas y preferidas por los autóctonos para los inmigrantes (Navas, Rojas, García, \& Pumares, 2007). Más en concreto, a los autóctonos se les pregunta, para el caso de las actitudes de aculturación plano ideal-, si prefieren que los inmigrantes vivan según la forma de vida de su país de origen o bien según las costumbres españolas, y también si prefieren que convivan con los españoles o prefieren que se relacionen entre ellos mismos; y para el caso de las estrategias o percepciones de aculturación -plano real- se les pregunta si creen que los inmigrantes viven según la forma de vida de sus países de origen o según las costumbres españolas, y también si creen que los inmigrantes 
conviven más con los españoles o creen que conviven más entre ellos. Por lo que se refiere a los inmigrantes, para el caso de las actitudes de aculturación -plano ideal- se les pregunta si preferirían vivir según la forma de vida de su país o según las costumbres españolas, y también si preferirían relacionarse más con los españoles o más entre ellos mismos; y para el caso de las estrategias -plano real- se les pregunta si realmente viven según la forma de vida de sus países o según las costumbres españolas, y también si realmente se relacionan más con los españoles o bien conviven más entre ellos. Según sea la combinación de respuestas entre ambas dimensiones se asignó a los participantes autóctonos e inmigrantes a una de las cuatro opciones de aculturación.

3. Test de multiculturalismo. Consta de 4 cuestiones obtenidas de las escalas de actitudes étnicas y multiculturales de Berry $\&$ Kalim (1995). El objetivo de esta escala era medir el grado de acuerdo o desacuerdo con la idea de una sociedad multicultural a través de las siguientes cuestiones: "Es beneficioso que haya diferentes grupos culturales conviviendo en un mismo país", "Me siento aceptado por los otros grupos culturales que me rodean", "Es una idea aceptable que personas de diferentes culturas se casen entre ellas" y "La diversidad cultural es una característica deseable para la sociedad mayoritaria”. El formato fue de respuesta tipo Likert de 5 puntos desde Muy de acuerdo a Muy en desacuerdo.

4. Escala de sensibilidad intercultural (Chen \& Starosta, 2000). Formada por un total de 24 ítems en formato tipo Likert de 5 puntos desde Muy de acuerdo a Muy en desacuerdo, encaminados a valorar la implicación, el respeto, la seguridad, el disfrute y la sensibilidad en las interacciones interculturales.

5. Test de Prejuicio: con el objetivo de comprobar la existencia de actitudes de prejuicio entre los participantes autóctonos hacia los inmigrantes, se incluyó el Test de Actitud Prejuiciosa (TAP) de Rojas, Navas, Lozano, \& Pérez (2011). Según estos autores, el TAP es una escala con adecuadas propiedades psicométricas que permite hacer comparaciones entre grupos y 
medir las peculiaridades de las actitudes sociales de prejuicio basadas en cogniciones o estereotipos, en emociones o afectos y en intenciones de conducta o distancia social preferida.

6. Índice de Aculturación de Vancouver (VIA) (Ryder, Alden, \& Paulhus, 2000). Consta de 20 ítems en formato de respuesta tipo Likert de 5 puntos desde Muy de acuerdo a Muy en desacuerdo. Este índice contempla un modelo bidimensional de aculturación a través de la medición de valores, relaciones sociales y adherencia a las tradiciones, tanto de la cultura de origen como de la de acogida.

\section{Resultados}

Según los resultados obtenidos en ambos estudios, una amplia mayoría de inmigrantes manifiestan actitudes de asimilación en su proceso de aculturación. En concreto, y teniendo en cuenta la media de ambos trabajos, $45 \%$ de los inmigrantes latinoamericanos residentes en la ciudad de Cuenca se decantan por la asimilación en su proceso de adaptación a la sociedad receptora. Aproximadamente, en torno a $70 \%$ de la muestra de autóctonos mostró preferencia por la separación como estrategia de aculturación para los inmigrantes. La opción de integración se constituyó en la segunda preferida para ambas muestras.

Las correlaciones obtenidas entre las variables utilizadas en ambos trabajos mencionados nos muestran una relación positiva entre la sensibilidad intercultural y el multiculturalismo en la muestra de inmigrantes, y también de estas variables con la preferencia por la cultura de acogida, medida a través de la escala VIA (Índice de Aculturación de Vancouver). Es decir, aquellos inmigrantes que se orientan en mayor medida hacia la cultura de acogida mostrarían mayor sensibilidad intercultural y tendencia hacia el multiculturalismo. De este resultado se desprende que aquellos inmigrantes con actitudes favorables hacia la cultura de origen y la cultura de acogida, mostrarían una clara sensibilidad intercultural y una tendencia al multiculturalismo entendido 
como actitud favorable hacia la convivencia entre culturas, sugiriendo una clara receptividad de los inmigrantes a mantener valores, relaciones y tradiciones de ambas culturas en su proceso de aculturación, lo que avala por tanto un modelo bicultural de adaptación sociocultural.

Con respecto a la muestra de autóctonos, cabe destacar que en esta la sensibilidad intercultural no se relacionó con ninguna de las variables restantes; sin embargo, el multiculturalismo se mostró relacionado con el prejuicio, de tal forma que las personas de la sociedad de acogida menos prejuiciosas se muestran más multiculturales.

Con el objetivo de ahondar en las posibles relaciones existentes entre las diferentes variables contempladas en este trabajo, se realizaron otros análisis bivariantes. En concreto, y con la muestra de inmigrantes, se realizaron ANOVAS contemplando las principales actitudes de aculturación como variables independientes y las diferentes escalas como variables dependientes. Hay que recordar, como se ha mencionado en el apartado de instrumento, que las actitudes de aculturación en cuanto variables independientes se generaron a partir del resultado obtenido de las variables dicotómicas obtenidas tras la combinación de respuestas a las dos preguntas sobre la importancia o no concedida a la conservación de la identidad cultural de origen y a la importancia o no de relacionarse con la sociedad de acogida. Los análisis realizados revelaron diferencias significativas en la escala de sensibilidad intercultural, de tal forma que los inmigrantes que prefieren la integración en su proceso de aculturación mostraron más sensibilidad intercultural que aquellos que prefieren la separación como opción de aculturación. También se hallaron diferencias significativas en la opción cultura de origen del Índice de Aculturación de Vancouver (VIA), de tal forma que los inmigrantes que optaron por la integración y la separación en su proceso de aculturación se mostraron más identificados con su cultura de origen que los que optaron por la asimilación. Por otro lado, los inmigrantes más jóvenes mostraron mayor actitud prejuiciosa que los inmigrantes de mayor edad.

Para el caso de los autóctonos, por medio de análisis $t$-test, considerando las actitudes de integración y asimilación como variables independientes y las escalas como variables dependientes, los resultados obtenidos mostraron diferencias significativas en la escala de 
multiculturalismo y de prejuicio. Los autóctonos que optaron por la integración como opción preferida de aculturación para los inmigrantes, se mostraron más favorables al multiculturalismo que los que eligieron la asimilación. Por otro lado, no se encontraron diferencias significativas en las actitudes de prejuicio entre aquellos sujetos que mostraron concordancia frente a los que mostraron discordancia entre sus preferencias y sus estrategias de aculturación; es decir, entre los que optaron por la misma o por diferente opción en los planos real e ideal. Por tanto, en este caso, al contrario que en los trabajos de Rohmann, Florack, \& Piontkowski (2006) y de Navas, Rojas, \& García (2011), no se hallaron diferencias significativas entre ambos grupos, ni en la muestra de inmigrantes ni tampoco en la de docentes.

Finalmente, por medio de análisis $t$-test para muestras independientes, se encontraron diferencias significativas en las puntuaciones obtenidas en la escala de sensibilidad intercultural y de prejuicio. Según las puntuaciones medias obtenidas por ambas muestras, los inmigrantes mostraron mayor grado de sensibilidad intercultural que los autóctonos y también mostraron menos actitud de prejuicio.

\section{Conclusiones}

Estos trabajos suponen un primer análisis sobre aculturación y sensibilidad intercultural en inmigrantes y autóctonos en un determinado contexto social específico. El principal objetivo ha consistido en estudiar las relaciones existentes entre la sensibilidad intercultural entendida como dimensión afectiva de la competencia comunicativa intercultural y las actitudes de aculturación y prejuicio en la población autóctona e inmigrante de la ciudad de Cuenca en España y siguiendo el marco teórico definido en los modelos de sensibilidad intercultural (Chen \& Starosta, 1996) y de aculturación (Berry, 1980).

Los resultados encontrados muestran que los inmigrantes prefieren la asimilación como opción de aculturación en su proceso de adaptación a la sociedad receptora, siendo la integración la segunda actitud elegida. Por su parte, la población autóctona también eligió 
la asimilación y la integración como las principales opciones de aculturación preferidas para los inmigrantes en su proceso de adaptación a la sociedad de acogida, aunque la muestra de docentes optó por la separación.

En estos trabajos, el proceso de aculturación se ha operativizado de forma general y no tanto por ámbitos de la vida y, al igual que en el trabajo de Navas, Rojas, \& García (2011), se constata también un considerable grado de desajuste entre las percepciones y las actitudes de aculturación de la población autóctona. Por contra, los resultados obtenidos en la muestra de inmigrantes reflejan un alto grado de concordancia entre sus preferencias y estrategias, dándose la mayor discrepancia en la integración como actitud y como estrategia, seguida de la marginación. Cabe mencionar que, en contra de los resultados ampliamente encontrados en la investigación sobre los procesos de aculturación, la integración como opción de aculturación o de adaptación sociocultural no se erige en la actitud o estrategia mayoritaria en las muestras utilizadas en los trabajos aquí referidos.

Sin embargo, los resultados obtenidos revelan que aquellos autóctonos que prefieren la integración manifiestan menos prejuicio que quienes optan por la asimilación, confirmando de este modo que los autóctonos e inmigrantes que optan por la opción de integración muestran menor nivel de prejuicio y coincidiendo así con estudios que encuentran hallazgos muy similares (Navas et al, 2006; Zick, Wagner, Van Dick, \& Petzel, 2001).

Con respecto a la sensibilidad intercultural, considerada como dimensión afectiva de la competencia comunicativa intercultural, los resultados muestran que los inmigrantes que prefieren la integración puntúan más alto en sensibilidad intercultural frente a los que eligen la separación. A su vez, los inmigrantes manifiestan mayor grado de sensibilidad intercultural que los autóctonos, lo que podría apuntar a un posible mayor grado de implicación, respeto y sensibilidad en las interacciones culturales.

Por otro lado, también entre los inmigrantes se encontraron altas correlaciones entre la sensibilidad y la identificación con la cultura de origen y con la de acogida, por ello la sensibilidad intercultural se mostró implicada en un posible modelo de competencia bicultural y 
de integración constructiva (Bennett, 1993; Bosky, 2008). De la misma forma, la sensibilidad intercultural se relacionó con el multiculturalismo en la muestra de inmigrantes, lo que podría estar indicando el respeto y sensibilidad de estos grupos de inmigrantes hacia otras culturas en general y hacia la cultura de acogida en particular. Estos datos obtenidos son coincidentes con los obtenidos en otros trabajos que contemplan la sensibilidad intercultural y el multiculturalismo como aspectos claramente implicados en la promoción de la diversidad cultural y en la superación del etnocentrismo (Berry \& Kalim, 1995; Chen \& Starosta, 2004).

Los resultados encontrados en estos trabajos de carácter exploratorio pueden considerarse como una primera contribución a este campo de estudio, poniendo de relieve la importancia futura de la dimensión aplicada de la sensibilidad intercultural basada en la implicación, respeto y disfrute de las relaciones culturales, como una importante variable para entender y fomentar la integración y adaptación sociocultural, la reducción de actitudes de discriminación y la superación del etnocentrismo, todo ello con el objetivo último de generar un modelo multicultural basado en la comprensión de los procesos de aculturación adecuados para la superación de conflictos, prejuicios y otras actitudes intergrupales negativas.

Finalmente, estos resultados deben ser considerados bajo algunas limitaciones metodológicas que hacen imposible su generalización y exigen cautela en su interpretación. Por un lado, en la medida en que las muestras utilizadas no son representativas de la población inmigrante ni tampoco de la población general de la ciudad, las conclusiones en ningún caso pueden generalizarse. En este sentido, cabe mencionar que no se consideró apropiado desagregar las muestras utilizadas por grupos en función de sus variables sociodemográficas debido a su escaso tamaño. A pesar de ello, los datos obtenidos con ambas muestras permiten considerar estos trabajos como un primer acercamiento a la relación existente entre la sensibilidad intercultural y los procesos de aculturación. Por otro lado, para la realización de estas investigaciones se elaboró un cuestionario específico integrado por una serie de escalas obtenidas expresamente para la consecución de los objetivos propuestos que, si bien en su conjunto han demostrado 
cierta validez, necesitan ser mejoradas para su uso como instrumentos con plena garantía de validez y fiabilidad. De la misma forma, con el objetivo de obtener resultados fiables y generalizables será necesario probar estas escalas con nuevas muestras obtenidas con procedimientos muestrales que permitan acceder a grupos de inmigrantes menos vinculados con organizaciones o asociaciones y también con muestras de autóctonos más representativas del conjunto de la población general, además de valorar posibles resultados según diferentes dominios sociales y ámbitos de vida.

La obtención de resultados generalizables, su comparación con otros estudios sobre actitudes interculturales y, consecuentemente, la generación de modelos de adaptación sociocultural aplicables a diversos contextos sociales, permitirán entender las relaciones intergrupales en las comunidades locales donde se manifiestan las actitudes y los comportamientos que emergen de las interacciones etnoculturales.

\section{Bibliografía}

Allport, G. W. (1954). The nature of prejudice. Reading, Mass: Addison-Wesley. Arango, J. (2000). Becoming a country of immigration at the end of the twentieth century: The case of Spain. En R. King, G. Lazaridis, \& C. Tsardanidis (Edits.), El dorado or Fortress Migration in Southern Europe (págs. 253276). London: MacMillan Press LTD.

Bennett, M. J. (1993). Towards ethnorelativism: A developmental model of intercultural sensitivity. En R. M. Paige (Ed.), Education for the intercultural experience. Yarmouth, ME: Intercultural Press.

Berry, J. W. (1980). Acculturation as varieties of adaptation. En A. M. Padilla (Ed.), Acculturation: Theory, models, and some new findings (págs. 9-25). Boulder, CO, Westview.

Berry, J. W. (1997). Immigration, acculturation and adaptation. Applied Psychology: An International Review, 46, 5-68.doi: 10.1111/j.14640597.1997.tb01087.x 
Berry, J. W., \& Kalim, R. (1995). Multicultural and ethnic attitudes in Canada: An overview of the 1991 national survey. Canadian Journal of Behavioral Science, 27, 301-320. doi: 10.1037/0008-400X.27.3.301

Bosky, P. (2008). Five meanings of integration in acculturation research. International Journal of Intercultural Relations, 32, 142-153. doi: 10.1016/j.ijintrel.2008.01.005

Bourhis, R. Y., Moïse, L. C., Perreault, S., \& Senécal, S. (1997). Towards and interactive acculturation model: a social psychology approach. International Journal of Psychology, 32, 369-386. doi: 10.1080/002075997400629

Briones, E.; Verkuyten, M.; Cosano, J. y Tabernero, C. (2012). Psychological adaptation of Maroccan and Ecuadorean immigrant adolescents in Spain. International Journal of Psychology, 47(1), 28-38. doi: 10.1080/00207594.2011.569722

Brown, R. (1995). Prejudice: its social psychology. Oxford, UK: Blackwell.

Chen, G. M. (1990). Intercultural communication competence: Some perspectives of research. The Howard of Communications, 2, 243-261. doi: 10.1080/10646179009359718

Chen, G. M. y Starosta, W. J. (1996). Intercultural communication competence: A synthesis. Communication Yearbook, 19, 353-384.

Chen, G. M. y Starosta, W. J. (2004). Communication among cultural diversities: A dialogue. International and Intercultural Comunication Annual, 27, 3-16.

Chen, G. y Starosta, W. J. (2000). The Development and validation of the Intercultural Sensitivity Scale. Reunión anual de la National Communication Association. Seatlle, WA.

Esses, V. M.; Wagner, U.; Wolf, C.; Preiser, M., y Wilbur, C. J. (2006). Perceptions of national identity and attitudes toward immigrants and immigration in Canada and Germany. International Journal of Intercultural Relations, 30, 653-669. doi:10.1016/j.ijintrel.2006.07.002

González, A. y Ramírez, M.P. (2016). La sensibilidad intercultural en relación con las actitudes de aculturación y prejuicio en inmigrantes y sociedad de acogida. Un estudio de caso. Revista Internacional de Sociología (RIS), 74(2), e034. doi: http://dx.doi.org/10.3989/ris.2016.74.2.034

Gudykunst, W. B., Wiseman, R. L. y Hammer, M. R. (1977). An analysis of an integrated approach to cross-cultural training. International Journal of Intercultural Relations, 1, 99-110. doi:10.1016/0147-1767(77)90045-1 
Hui, B. P., Chen, S. X., Leung, C. M., \& Berry, J. W. (2016). Facilitating adaptation and intercultural contact: The role of integration and multicultural ideology in dominant and non-dominant groups. International Journal of Intercultural Relations, 45, 70-84.

International Organization for Migration. (2012). Recuperado el 30 de 12 de 2012, de http://www.iom.int

Kosic, A., Mannetti, L. y Sam, D. L. (2005). The role of majority attitudes towards outgroup in the perceptions of the acculturation strategies of immigrants. International Journal of Intercultural Relations, 29, 273288. doi: 10.1016/j.ijintrel.2005.06.004

Labedeva, N., Tatarko, A. y Berry, J. W. (2016). Intercultural relations among migrants from Caucasus and Russians in Moscow. International Journal of Intercultural Relations, 52, 27-38.

Navas, M., García, M. C., Rojas, A. J., Pumares, P., \& Cuadrado, I. (2006). Actitudes de aculturación y prejuicio: la perspectiva de autóctonos e inmigrantes. Psicothema, 18, 187-193.

Navas, M.; García, M. C.; Sánchez, J.; Rojas, A. M., Pumares, P. y Fernández, J. S. (2005). Relative Acculturation Extended Model (RAEM): New contributions with regard to the study of acculturation. International Journal of Intercultural Relations, 29, 21-37. doi:10.1016/j.ijintrel.2005.04.001

Navas, M., Rojas, A. J. y García, M. C. (2011). Concordancia entre actitudes y percepciones de aculturación de la población autóctona hacia los inmigrantes magrebíes: relación con las actitudes prejuiciosas. Anales de psicología, 27, 186-194.

Navas, M.; Rojas, A. J.; García, M. C. y Pumares, P. (2007). Acculturation strategies and attitudes according to the Relative Acculturation Extended Model (RAEM): The perspectives of natives versus immigrants. International Journal of Intercultural Relations, 31, 67-86. doi: 10.1016/j.ijintrel.2006.08.002

Padilla, A. M. (1980). The role of cultural awareness and ethnic loyality in acculturation. En A. M. Padilla (Ed.), Theory, models and some new findings (págs. 47-84). Boulder, CO: Westview.

Padilla, A. M. y Perez, W. (2003). Acculturation, social identity, and social cognition: a new perspective. Hispanic Journal of Behavioral Sciences, 25(1), 35-55. doi: 10.1177/0739986303251694 
Pfafferott, I. y Brown, R. (2006). Acculturation preference of majority and minority adolescents in Germany in the context of society and family. International Journal of Intercultural Relations, 30, 703-717. doi: 10.1016/j.ijintrel.2006.03.005

Piontkowski, U.; Rohmann, A. y Florack, A. (2002). Concordance of acculturation attitudes and perceived threat. Group Processes \& Intergroup Relations, 5, 221-232. doi: 10.1177/1368430202005003003

Portes, A., Celaya, A., Vickstrom, E. y Aparicio, R. (2012). Who are we? Parental influences on self-identities and self-esteem on second Generation youths in Spain. Revista Internacional de Sociología (RIS), 70(1), 9-37. doi: 10.3989/ris.2011.09.26

Redfield, R., Linton, R. y Herskovits, M. J. (1936). Memorandum on the study of acculturation. American Anthropology, 38, 149-152. doi: 10.1525/ aa.1936.38.1.02a00330

Rodríguez-García, D. (2010). Beyond assimilations and multiculturalism: a critical review of the debate on managing diversity. International Migration and Integration, 11, 251.271. doi: 10.1007/s12134-010-0140-x

Rohmann, A., Florack, A. y Piontkowski, U. (2006). The role of discordamt acculturation attitudes in perceived threat: An analysis of host and immigrant attitudes in Germany. International Journal of Intercultural Relations, 30, 683-702. doi:10.1016/j.ijintrel.2006.06.006

Rojas, A.; Navas, M.; Lozano, O. M. y Pérez, P. J. (2011). Prejudiced attitude measurement using the rasch rating scale model. Psychology Reports, 109, 553-572. doi: 10.2466/07.17.PR0.109.5.553-572

Ruben, B. D. y Kealy, D. J. (1979). Behavioural assessment of communication competency and the prediction of cross-cultural adaption. International Journal of Intercultural Relations, 3, 15-48. doi: 10.1016/0147-1767(79)90045-2

Rudmin, F. W. (2009). Constructs, measurements and models of acculturation and acculturative stress. International Journal of Intercultural Relations, 33, 106-123. doi: 10-1016/j.ijintrel.2008.12.001

Ryder, A. G.; Alden, L. E. y Paulhus, D. L. (2000). Is acculturation unidimensional or bidimensional? A head-to-head comparasion in the prediction of personality, self-identify, and adjustment. Journal of Personality and Social Psychology, 79(1), 49-65. doi:10.1037/0022-3514.79.1.49 
Van Oudenhoven, J. P., Prins, K. S. y Buunk, B. P. (1998). Attitudes of minority and majority members toward adaptation of immigrants. European Journal of Social Psychology,28,995-1013.doi: 10.1002/ (SICI)1099-0992(1998110)28:6<995:AID-EJSP908>3.0.CO;2-8

Ward, C. y Kennedy, A. (1994). Acculturation strategies, psuchological adjustment, and sociocultural competence during cross-cultural transitions. International Journal of Intercultural Relations, 18(3), 329-343. doi: 10.1016/0147-1767(94)90036-1

Zagefka, H. y Brown, R. (2002). The relationship between acculturation strategies, relative fit and intergroup relations: Immigrant-majority relations in Germany. European Journal of Social Psychology, 32, 171-188.

Zick, A.; Wagner, U.; Van Dick, P. y Petzel, T. (2001). Acculturation and prejudice in Germany: majority and minority perspectives. Journal of Social Issues, 57, 541-557. doi: 10.1111/0022-4537.00228 